\title{
Why dimensional analysis is irrational, and correlations based on it are suspect.
}

\author{
Eugene F. Adiutori \\ efadiutori@aol.com \\ 239-537-4107 \\ Ventuno Press \\ 1094 Sixth Lane N. \\ Naples, FL 34102 \\ November 5, 2021
}

\begin{abstract}
The purpose of dimensional analysis is to minimize the number of parameters that must be varied in an experiment designed to generate data for a generic correlation. Dimensional analysis requires that parameter dimensions be multiplied and divided in order to identify dimensionless groups of parameters in correlations. Since the number of dimensionless groups in correlations is generally smaller than the number of parameters, the number of parameters that must be varied in the experiment is reduced. The experiment generates the data required to quantify coefficients and exponents in correlations. Because dimensional analysis requires that dimensions be multiplied and divided, it is rational only if it is rational to multiply and divide dimensions. From the beginning of science until 1822, scientists and engineers such as Galileo, Hooke, and Newton agreed that dimensions cannot rationally be multiplied or divided, and therefore equations cannot rationally describe how parameters are related. In 1822, Fourier made the revolutionary and unproven claims that dimensions can rationally be assigned to numbers, and dimensions can rationally be multiplied or divided. Thereafter, equations could rationally describe how parameters are related. Even though Fourier's claims have never been proven, they are fundamental tenets in modern engineering science. However, critical appraisal in the text proves that Galileo, Hooke, and Newton were correct, and Fourier was wrong. Dimensions cannot rationally be multiplied and divided. Consequently dimensional analysis is irrational, and correlations based on dimensional analysis are suspect because the functionalities attributed to unvaried parameters are not supported by data. Those functionalities are supported only by the erroneous assumption that dimensions can rationally be multiplied and divided.
\end{abstract}

\section{Introduction}

The purpose of dimensional analysis is to minimize the number of parameters that must be varied in experiments to determine generic correlations. Because dimensional analysis requires the multiplication and division of dimensions, it is rational only if it is rational to multiply or divide dimensions. This article critically appraises the multiplication and division of dimensions in order to determine whether or not dimensional analysis is rational, and whether or not correlations based on dimensional analysis accurately describe behavior.

\section{Dimensional analysis}

The purpose of dimensional analysis is to reduce the number of parameters that must be varied in an experiment designed to result in a generic and dimensionless correlation. Dimensional analysis requires that parameter dimensions be multiplied and divided in order to deduce 
dimensionless groups of parameters that replace individual parameters in correlations. The experiment provides the data required to quantify coefficients and exponents in correlations. Since the number of dimensionless groups in correlations is generally smaller than the number of parameters, dimensional analysis reduces the number of parameters that must be varied in experiments.

\section{Until 1822, scientists and engineers agreed that, with one exception, dimensioned parameters cannot be multiplied or divided.}

Until the publication of Fourier's The Analytical Theory of Heat" [1] in 1822, scientists and engineers such as Galileo, Hooke, and Newton agreed that:

- Parameter symbols in equations represent numerical value and dimension.

- Equations must be dimensionally homogeneous.

- With one exception, dimensioned parameters cannot be multiplied or divided.

- The one exception is that a dimensioned parameter can be divided by the same dimensioned parameter. For example, 10 meters can be divided by 5 meters resulting in 2, and 6 seconds can be divided by 12 seconds resulting in 0.5 , but 9 meters cannot be divided by 15 seconds resulting in 0.6 (meters divided by seconds) because meters cannot rationally be divided by seconds.

- Because dimensioned parameters cannot be multiplied, and because only like dimensioned parameters can be divided, rational parametric equations must include only ratios of like parameters. The following dimensionally homogeneous verbal equation by Galileo is typical.

If two particles are carried at a uniform rate, the ratio of their speeds will be the product of the ratio of the distances traversed by the inverse ratio of the time-intervals occupied.

Galileo [2]

Galileo's equation includes the ratios speed to speed, distance traversed to distance traversed, and time-interval to time-interval. Because the ratios are pure numbers, they can be multiplied or divided.

Galileo and his contemporaries saw no conflict between the concept of speed, and the view that distance cannot be divided by time. To Galileo and his contemporaries, distance and time are necessary to quantify speed, but speed has nothing to do with dividing distance by time. In Galileo's view, and in the view of the science community from the beginning of science until the year 1822, dividing distance by time is irrational.

\section{Why Hooke's law [3], Newton's law of cooling by Newton [4], and Newton's second law of motion by Newton [5] were not equations.}

Because proportions that relate two parameters do not require that dimensions be multiplied or divided, proportions were generally used instead of equations until 1822. That is why 
Hooke's law [3] is Proportion (1), Newton's law of cooling by Newton [4] is Proportion (2) ${ }^{1}$, and Newton's second law of motion by Newton [5] is Proportion (3).

$\sigma \alpha \varepsilon$

$\left(\mathrm{dT}_{\text {body }} / \mathrm{dt}\right) \alpha\left(\mathrm{T}_{\text {body }}-\mathrm{T}_{\text {ambient }}\right)$

a $\alpha \mathrm{f}$

5. The only reason it is now considered rational to multiply or divide dimensions.

The only reason it is now considered rational to multiply or divide dimensions is because in 1822, Fourier's [3] treatise The Analytical Theory of Heat proposed a revolutionary and unproven view of dimensional homogeneity in which dimensions can rationally be assigned to numbers, and dimensions can rationally be multiplied and divided. Fourier's treatise describes his revolutionary and unproved view of dimensional homogeneity in the following:

... every undetermined magnitude or constant has one dimension proper to itself, and the terms of one and the same equation could not be compared if they had not the same exponent of dimensions. (This view of dimensional homogeneity) is the equivalent of the fundamental lemmas (axioms) which the Greeks have left us without proof.

In his nearly 500 page treatise, Fourier made no effort to prove that dimensions can rationally be assigned to numbers, or that dimensions can rationally be multiplied and divided. Presumably Fourier's colleagues accepted his unproven views because they enabled him to solve problems that had never been solved.

From data he obtained, Fourier concluded that, if a warm, solid body is cooled by the steadystate forced convection of ambient air, the relationship between $\mathrm{q}$ and $\Delta \mathrm{T}$ is always described by Eq. (4) in which $c$ is the constant of proportionality, $q$ is the numerical value and dimension of heat flux, and $\Delta \mathrm{T}$ is the numerical value and dimension of temperature difference.

$\mathrm{q}=\mathrm{c} \Delta \mathrm{T}$

Fourier's unproven view that dimensions can rationally be assigned to numbers made it rational for him to create dimensioned parameter $h$ from pure number $c$ by assigning it the dimension of $(q / \Delta T)$. His unproven view that dimensions can rationally be multiplied or divided made it rational for him to create Eq. (5), the first of many laws of conventional engineering science that were created by applying Fourier's revolutionary and unproven views that dimensions can rationally be assigned to numbers, and dimensions can rationally be multiplied and divided.

$\mathrm{q}=\mathrm{h} \Delta \mathrm{T}$

To Fourier and his colleagues, Eq. (5) was a law, and the law meant "If heat transfer is by steady-state forced convection of ambient air flowing over a warm solid body, the numerical

\footnotetext{
${ }^{1}$ American heat transfer texts generally refer to the equation $\mathrm{q}=\mathrm{h} \Delta \mathrm{T}$ as "Newton's law of cooling", but this equation cannot be Newton's law of cooling because cooling is transient behavior, and the equation is a steadystate equation. Note that Proportion (2) describes transient behavior.
} 
value and dimension of convection heat flux always equal the numerical value and dimension of heat transfer coefficient times the numerical value and dimension of temperature difference, and $\mathrm{h}$ is always the numerical value and dimension of the constant of proportionality".

Even though Fourier's revolutionary claims (that dimensions can rationally be assigned to numbers, and dimensions can rationally be multiplied or divided) have never been validated, they are generally considered fundamental tenets in modern engineering science.

\section{Proof that dimensions cannot rationally be multiplied and divided.}

"Multiply four times six" means "add six four times". Therefore "multiply meters times kilograms" must mean "add kilograms meters times". Because "add kilograms meters times" has no meaning, dimensions cannot be multiplied.

"Divide twenty by five" means "how many fives are in twenty". Therefore "divide meters by seconds" must mean "how many seconds are in meters". Because "how many seconds are in meters" has no meaning, dimensions cannot be divided.

\section{Q. E. D. Dimensions cannot be multiplied or divided.}

\section{Conclusions}

- Dimensional analysis is irrational because it requires that dimensions be multiplied and divided when in fact dimensions cannot rationally be multiplied and divided.

- Correlations based on dimensional analysis are highly suspect because the functionalities assigned to parameters that were not varied in underlying experiments are not supported by data. They are supported only by the erroneous assumption that dimensions can rationally be multiplied and divided.

\section{Symbols}

$\begin{array}{ll}\text { a } & \text { acceleration } \\ \mathrm{f} & \text { force } \\ \mathrm{h} & \text { heat transfer coefficient } \\ \mathrm{q} & \text { heat flux } \\ \mathrm{T} & \text { temperature } \\ \mathrm{t} & \text { time } \\ \varepsilon & \text { strain } \\ \sigma & \text { stress }\end{array}$

\section{References}

[1] Fourier, J., 1822, The Analytical Theory of Heat, 1955 Dover edition of 1878 English translation, Article 160

[2] Galileo, Concerning Two New Sciences, 1638, p. 199, Encyclopedia Britannica, 1952

[3] Hooke, R., 1676, encoded in "A Description of Helioscopes", per Robert Hooke's Contributions to Mechanics by F. F. Centore, Nartinus Nijhoff/The Hague, 1970

[4] Newton, I., 1701, “A Scale of the Degrees of Heat”, Phil Trans Royal Soc (London), 22, p. 824 
[5] Newton, I., 1726, The Principia, $3^{\text {rd }}$ edition, translation by Cohen, I. B. and Whitman, A. M., 1999, University of California Press, p. 460

[6] Fourier, J., 1822, The Analytical Theory of Heat, 1955 Dover edition of 1878 English translation, Article 36 